\title{
Should lung scan be abandoned for pulmonary embolism diagnosis in the age of multislice spiral CT? No
}

\author{
Massimo Miniati · Simonetta Monti
}

Received: 4 March 2009/Accepted: 24 March 2009/Published online: 28 April 2009

(C) SIMI 2009

Multidetector computed tomographic angiography (CTA) is increasingly recognized as the first-line imaging technique for suspected pulmonary embolism [1], however, CTA is not always feasible. In the second Prospective Investigation of Pulmonary Embolism Diagnosis (PIOPED II), over $50 \%$ of the 7,284 eligible patients were excluded for documented contraindications to CTA such as renal failure, abnormal creatinine levels, allergy to contrast agents, critical illness requiring mechanical ventilation, recent myocardial infarction, or pregnancy [2]. Alternative imaging protocols are therefore needed.

Perfusion scintigraphy was introduced in the 1960s to visualize the regional distribution of pulmonary blood flow. Some early studies showed that segmental or lobar perfusion defects on lung scintigraphy are characteristic of acute pulmonary embolism [3, 4].

In the 1970s, ventilation imaging was added to perfusion scintigraphy on the assumption that it could help differentiate perfusion defects due to embolism-where ventilation ought to be relatively preserved-from perfusion abnormalities secondary to disorders of the lung parenchyma where both ventilation and perfusion are impaired. Contrary to expectations, ventilation-perfusion scintigraphy often yields inconclusive results, especially when obsolete ventilation tracers (e.g. 133-Xenon) are used [5]. In recent years, the introduction of newer ventilation

M. Miniati $(\bowtie)$

Dipartimento di Area Critica Medico Chirurgica,

Università degli Studi di Firenze,

Viale Morgagni 85, 50134 Florence, Italy

e-mail: massimo.miniati@unifi.it

S. Monti

Istituto di Fisiologia Clinica del Consiglio Nazionale delle

Ricerche, Pisa, Italy tracers (e.g. Technegas ${ }^{\circledR}$ ) and the implementation of single photon emission tomography have led to a substantial improvement in the diagnostic accuracy [6]. Yet, ventilation imaging cannot be obtained in critically ill patients. Moreover, ventilation-perfusion scintigraphy carries a higher radiation load than perfusion scanning alone, which makes it inappropriate in evaluating young subjects or pregnant women with suspected pulmonary embolism.

The diagnostic value of perfusion scintigraphy (without ventilation imaging) was reappraised in the Prospective Investigative Study of Acute Pulmonary Embolism Diagnosis (PISAPED) published in 1996 [7]. In that study, a lung scan featuring wedge-shaped (segmental) perfusion defects is diagnostic with a sensitivity of $86 \%$ and a specificity of $93 \%$ against selective or superselective pulmonary angiography [7]. These values are in close agreement with those reported for multidetector CTA in the PIOPED II (sensitivity $83 \%$, specificity $96 \%$ ). The diagnostic performance of perfusion scintigraphy for lung embolism was confirmed upon examining 889 scans from the PIOPED II [8]. The PISAPED criteria for interpreting perfusion scans have been recently reviewed [9]. An example of a perfusion scan suggestive of acute pulmonary embolism is given in Fig. 1 .

It appears, therefore, that perfusion scintigraphy is as accurate as a multidetector CTA in diagnosing or excluding lung embolism. Differently from CTA, it is essentially norisk, minimally invasive, and requires no iodine injection. In addition, it is less expensive and entails a much lower radiation burden than $\mathrm{CTA}$.

As said, it should be remembered that none of the available diagnostic tests for suspected pulmonary embolism can, alone, safely confirm or exclude the diagnosis without independent assessment of the clinical probability of the disease. The latter can be expressed empirically or by means of a prediction rule $[10,11]$. 


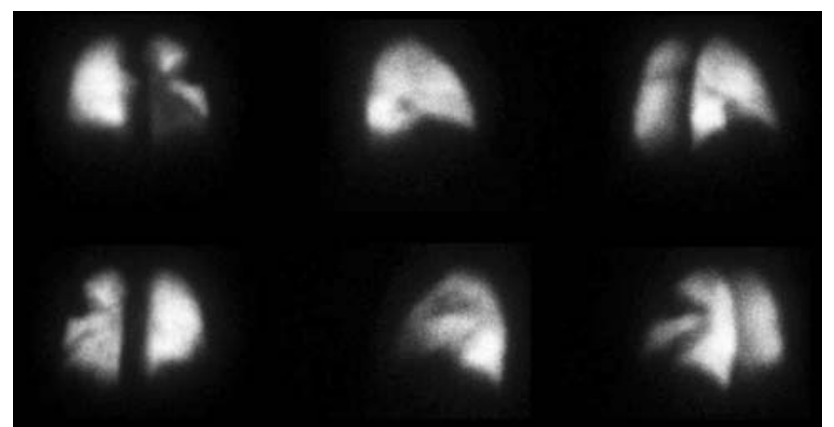

Fig. 1 Perfusion scintigraphy of a patient with acute pulmonary embolism. Images (from top left to bottom right) are: anterior, right lateral, right posterior oblique, posterior, left lateral, left posterior oblique. Multiple wedge-shaped perfusion defects are evident in both lungs

Considering the sensitivity and specificity of perfusion scintigraphy, the following statements can be made: (a) a normal perfusion scan rules out pulmonary embolism, (b) a perfusion scan with segmental perfusion defects, paired with a clinical probability $>50 \%$, makes the diagnosis of pulmonary embolism very likely and justifies anticoagulant therapy; (c) a perfusion scan featuring perfusion defects other than segmental with a low $(<10 \%)$ clinical probability makes the diagnosis of pulmonary embolism very unlikely and justifies withholding anticoagulation; (d) when clinical probability and results of lung scanning are discordant, the post-test probability is neither sufficiently high nor sufficiently low to permit therapeutic decisions. Under these circumstances further testing, such as lower limb compression ultrasonography or CTA, is required to confirm or exclude the diagnosis.

A strategy based on combining standardized clinical probability with perfusion scintigraphy permits a noninvasive diagnosis or exclusion of pulmonary embolism in $83 \%$ of patients, and appears to be safe because patients who are deemed not to have pulmonary embolism have a very low $(0.4 \%)$ thromboembolic risk over a 1-year period of follow up [12].

Because of its inherent characteristics, perfusion scintigraphy is particularly suitable to assess changes in regional pulmonary blood flow over time-a fundamental issue when dealing with patients diagnosed as having pulmonary embolism. Monitoring the resolution of pulmonary emboli by perfusion scintigraphy is instrumental in evaluating the efficacy of anticoagulant therapy, and to promptly identify patients with persistent perfusion abnormalities who may be at risk of developing chronic thromboembolic pulmonary hypertension [13].

Conflict of interest statement The authors declare that they have no conflict of interest related to the publication of this manuscript.

\section{References}

1. Tapson VF (2008) Acute pulmonary embolism. N Engl J Med 358:1037-1052

2. Stein PD, Fowler SE, Goodman LR et al (2006) Multidetector computed tomography for acute pulmonary embolism. N Engl J Med 354:2317-2327

3. Gilday DL, James AE Jr (1972) Lung scan patterns in pulmonary embolism versus those in congestive heart failure and emphysema. Am J Roentgenol Radium Ther Nucl Med 115:739-750

4. Moses DC, Silver TM, Bookstein JJ (1974) The complementary roles of chest radiography, lung scanning, and selective pulmonary angiography in the diagnosis of pulmonary embolism. Circulation 49:179-188

5. PIOPED Investigators (1990) Value of the ventilation-perfusion scan in acute pulmonary embolism: results of the prospective investigation of pulmonary embolism diagnosis (PIOPED). JAMA 263:2753-2759

6. Bajc M, Olsson B, Palmer J et al (2008) Ventilation/Perfusion SPECT for diagnostics of pulmonary embolism in clinical practice. J Intern Med 265:379-387

7. Miniati M, Pistolesi M, Marini C et al (1996) Value of perfusion lung scan in the diagnosis of pulmonary embolism. Results of the prospective investigative study of acute pulmonary embolism diagnosis (PISA-PED). Am J Respir Crit Care Med 154:13871393

8. Sostman HD, Miniati M, Gottschalk A et al (2008) Sensitivity and specificity of perfusion scintigraphy combined with chest radiography for acute pulmonary embolism in PIOPED II. J Nucl Med 49:1741-1748

9. Miniati M, Sostman HD, Gottschalk A et al (2008) Perfusion lung scintigraphy for the diagnosis of pulmonary embolism: reappraisal and review of the PISAPED method. Sem Nucl Med $38: 450-461$

10. Miniati M, Monti S, Bottai M (2003) A structured clinical model for predicting the probability of pulmonary embolism. Am J Med 114:173-179

11. Miniati M, Bottai M, Monti S et al (2008) Simple and accurate prediction of the clinical probability of pulmonary embolism. Am J Respir Crit Care Med 178:290-294

12. Miniati M, Monti S, Bauleo C et al (2003) A diagnostic strategy for pulmonary embolism based on standardized pretest probability and perfusion lung scanning-A management study. Eur J Nucl Med Mol Imag 30:1450-1456

13. Miniati M, Monti S, Bottai M et al (2006) Survival and restoration of pulmonary perfusion in a long-term follow-up of patients after acute pulmonary embolism. Medicine (Baltimore) 85:253262 